\title{
Indications for reoperation after sleeve gastrectomy
}

\author{
๑ Fadli Doğan,' ๑ Mürşit Dinçer² \\ 'Department of General Surgery, Elazığ Medical Park Hospital, Elazığ, Turkey \\ ${ }^{2}$ Department of General Surgery, Fırat University Faculty of Medicine, Elazı̆̆, Turkey
}

\begin{abstract}
Introduction: Morbid obesity is a serious health condition. Surgical interventions play an essential role in treatment, and sleeve gastrectomy is one of the most widely performed bariatric surgical procedures. After bariatric surgery, some patients may require reoperation for several reasons, such as complications, cholelithiasis, and gastroesophageal reflux. This study is an examination of the indications for reoperation and the results observed in patients who underwent reoperation following sleeve gastrectomy.

Materials and Methods: Patients who were reoperated on after sleeve gastrectomy for various reasons were analyzed retrospectively. The demographic characteristics, reoperation indications, surgical procedures, and results were analyzed.

Results: Of a total of 1268 patients who underwent sleeve gastrectomy due to obesity, 15 who had a reoperation after laparoscopic sleeve gastrectomy were included in the study. The overall reoperation rate after sleeve gastrectomy was $1.18 \%$. Cholelithiasis leak, hemorrhage, stenosis, intra-abdominal hematoma, and incisional hernia were identified as indications for reoperation.

Conclusion: Reoperation for the patients who undergo bariatric surgery may be required for a number of reasons. The results of this study indicate that a second procedure after sleeve gastrectomy can be performed safely and with minimal risk.

Keywords: Bariatric surgery; morbid obesity; sleeve gastrectomy.
\end{abstract}

\section{Introduction}

Morbid obesity is one of the most serious diseases in the world. ${ }^{[1-3]}$ Morbid obesity refers to body mass index (BMI) levels of $40 \mathrm{~kg} / \mathrm{m}^{2}$ or higher. ${ }^{[4]}$ Morbid obesity is a risk factor for many systemic diseases such as diabetes, cardiopulmonary diseases and deep vein thrombosis. ${ }^{[5]}$ The effectiveness of diet, exercise and medical treatments are limited for the treatment of morbid obesity. Bariatric surgery is becoming increasingly important for the treatment of morbid obesity. ${ }^{[3,6]}$ Roux-en-Y Gastric Bypass (RYGB) is a safe procedure that has proven its effectiveness for the treatment of morbid obesity for many years. Due to its technical difficulties, alternative surgical procedures to RYGB have been developed recently. Laparoscopic sleeve gastrectomy (LSG), which was previously used as a part of biliopancreatic diversion surgeries, has become a surgical procedure that is now performed alone for the treatment of morbid obesity. ${ }^{[2]}$ It is getting more popular because there is no anastomosis construction and it is easier to apply than the other procedures. Despite the low rates of complications, complications such as bleeding and leak may be seen at 
times. Some of these complications can only be controlled by reoperation. This study aims to present the results of the cases that were reoperated after sleeve gastrectomy.

\section{Materials and Methods}

The patients, who were followed-up for morbid obesity at Elazığ Medical Park Hospital General Surgery Clinic between January 2016 and February 2019, were investigated retrospectively. The patients, who had surgical indications and underwent sleeve gastrectomy, were evaluated through the hospital database. The cases who were not reoperated after sleeve gastrectomy were excluded from the study (Fig. 1). The patients who were reoperated for various reasons after sleeve gastrectomy were included in the study (Fig. 2). Demographic characteristics of the patients, indications and results of reoperation were analyzed.

Ethics committee approval and informant consent were not obtained due to the retrospective design of the study. In addition to that, the study was carried out in accordance with the ethical principles of the World Medical Association's Declaration of Helsinki.

\section{Statistics}

Statistical analysis was performed by using the SPSS Statistics software package, version 20 (IBM Corp. in Ar-

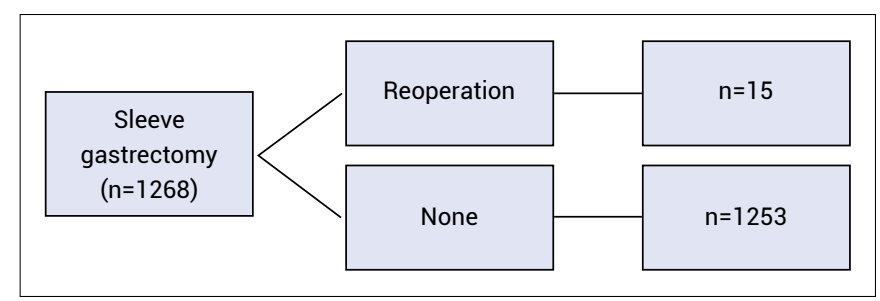

Figure 1. Incidence of reoperation after sleeve gastrectomy.

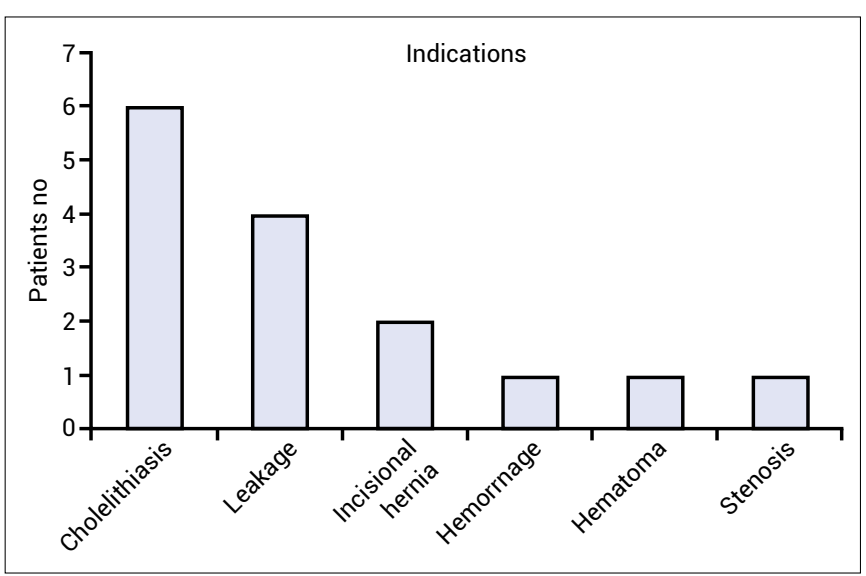

Figure 2. Indications of reoperation. monk, NY). Descriptive data were presented as frequencies (n) and percentages (\%) for categorical variables and as mean with standard deviation or median with (min-max range). for non-normal distributed numerical variables.

\section{Results}

The patients, who underwent sleeve gastrectomy for morbidity obesity in Elazığ Medical Park Hospital between December 2016 and February 2019, were investigated retrospectively through the hospital database. One thousand two hundred and sixty eight patients were determined who underwent sleeve gastrectomy due to obesity. Patients who were followed up conservatively, who had interventional radiology procedures or underwent endoscopic stenting without surgical drainage were excluded from the study. Fifteen cases that were reoperated after Laparoscopic Sleeve Gastrectomy (LSG) were included in the study. Overall reoperation rate after sleeve gastrectomy was $1.18 \%$. Seven of the patients were female and eight of them were male. The mean age was $37.26( \pm 7.95)$. The mean BMI of reoperated patients was $45.67( \pm 3.64)$. One case was reoperated due to bleeding in the 6 th hour postoperatively. Surgical drain was placed in a patient due to intra-abdominal fluid collection on the 6th day postoperatively. Catheter was inserted surgically in 4 of the 10 cases who developed leak. In 3 of these cases, leaks were closed after conservative follow-up and one of the cases required endoscopic stenting. RYGB procedure was performed to the patient who developed stenosis after sleeve gastrectomy. Two cases were reoperated due to incisional hernia. Six cases underwent laparoscopic cholecystectomy due to symptomatic cholelithiasis (Table 1). No additional intervention was required for other cases except the case who had endoscopic stenting after the leak. No complications were observed related to the second operations.

\section{Discussion}

Sleeve gastrectomy is one of the most widely performed procedures in bariatric surgery. The similar surgical results compared to the other techniques, low complication rates and relatively easier application make this technique effective and reliable. ${ }^{[7]}$ The overall complication rate in large medical centers is $\left\langle 15 \%{ }^{\left[{ }^{[8]}\right.}\right.$ Khoursheed et al. ${ }^{[9]}$ reported their overall complication rate was $7.5 \%$. The most serious complications after sleeve gastrectomy are bleeding and leak. ${ }^{[8]}$ Bleeding ratio after sleeve gastrectomy is 1 to $2 \%$. Some patients may require blood transfusion and/ or re-exploration. ${ }^{[9]}$ Khoursheed et al. ${ }^{[9]}$ reported reopera- 
Table 1. Management of patients

\begin{tabular}{lccc} 
Indications & Patients, $\mathbf{n}(\%)$ & Surgical procedures & Additional intervention \\
\hline Cholelithiasis & $6(40)$ & Laparoscopic cholecystectomy & None \\
Leakage & $4(26.6)$ & Drainage & Endoscopic stent $(\mathrm{n}=1)$ \\
Incisional hernia & $2(13.3)$ & Repair & None \\
Stenosis & $1(6.6)$ & RYGB & None \\
Hemorrhage & $1(6.6)$ & Hemostasis & None \\
Intraabdominal hematoma & $1(6.6)$ & Drainage & None \\
\hline RYGB: Roux-en-Y Gastric Bypass. & & & \\
\hline
\end{tabular}

tion rate as $0.5 \%$ due to bleeding. In this study, one case was reoperated due to bleeding in the 6th hour postoperatively. Surgical drain was placed in a patient due to intraabdominal fluid collection on the 6th day postoperatively. Postoperative staple line leak was reported to occur in $0-6.7 \%$ of patients in systematic reviews. ${ }^{[9]}$ Catheter was inserted surgically in 4 patients who developed leak. In 3 of these cases, leaks were closed after conservative followup and a case required endoscopic stenting.

The leak rates after sleeve gastrectomy were reported in various rates in the literature, but they are generally low. [8,10,1] Sleeve Gastrectomy Expert Panel Consensus Statement 2011, the leak rate was $1.06 \%{ }^{[12]}$ Surgical intervention may be necessary in cases that cannot be conducted conservatively. The overall leak rate was observed to be $0.78 \%$ in our study. Surgical catheter was inserted in $40 \%$ of the cases that developed a leak and were followed up conservatively. No major revision surgery was performed in any case due to leak. One case was required endoscopic stent procedure after surgical drain catheterization.

There is no clear information in the literature about the incidence of cholelithiasis after sleeve gastrectomy. Rapid weight loss after bariatric surgery is regarded as responsible for the formation of cholelithiasis. ${ }^{[13]}$ The incidence of symptomatic gallstones requiring cholecystectomy after laparoscopic sleeve gastrectomy (LSG) was reported as $0.9-7.5 \%$ in the literature. ${ }^{[13]}$ In follow-ups of the 1268 cases operated in this study, symptomatic cholelithiasis was detected in 6 cases $(0.47 \%)$. Cholelithiasis was not known preoperatively in these patients. The earliest case was in the $4^{\text {th }}$ month and the latest case was in the $3^{\text {nd }}$ year.

In the literature, approximately $5-10 \%$ of SG patients have been reported to require revision procedures. ${ }^{[7]}$ Yorke et al. ${ }^{[7]}$ reported conversion rates as $6.6 \%$. RYGB procedure was performed to one patient who developed stenosis after sleeve gastrectomy in this study (0.07\%). Revision rates are low compared with the literature. However, no patients underwent revision because of insufficient weight loss.

While morbid obesity causes many systemic diseases, it significantly reduces the surgical risk for the patient group who need elective surgery. After bariatric surgery, some of the patients can be reoperated due to complications such as cholelithiasis, gastroesophageal reflux. This study reveals that additional surgical interventions can be performed safely after sleeve gastrectomy.

There are some limited aspects of this study. The retrospective design of the study and the limited number of reoperated patients are the limited aspects of this study. However, when we look at the literature, the results are similar to this study in terms of the reoperation rates.

\section{Conclusion}

Morbid obesity is one of the most serious diseases. Sleeve gastrectomy has become the most commonly used procedure for the treatment of morbid obesity. Some patients need additional surgical procedures after sleeve gastrectomy. Additional surgical procedures can be performed safely for these patients.

\section{Disclosures}

Financial Support: No financial support was received in this study.

Ethichs Committee Approval: Ethics committee approval was not obtained because it was a retrospective study.

Peer-review: Externally peer-reviewed.

Conflict of Interest: None declared. 


\section{References}

1. Iannelli A, Dainese R, Piche T, Facchiano $E$, Gugenheim J. Laparoscopic sleeve gastrectomy for morbid obesity. World J Gastroenterol 2008;14:821-7. [CrossRef]

2. Benaiges D, Más-Lorenzo A, Goday A, Ramon JM, Chillarón JJ, Pedro-Botet J, et al. Laparoscopic sleeve gastrectomy: More than a restrictive bariatric surgery procedure? World $\mathrm{J}$ Gastroenterol 2015;21:11804-14. [CrossRef]

3. Kang JH, Le QA. Effectiveness of bariatric surgical procedures: A systematic review and network meta-analysis of randomized controlled trials. Medicine (Baltimore) 2017;96:e8632. [CrossRef]

4. Lee WJ, Yu PJ, Wang W, Chen TC, Wei PL, Huang MT. Laparoscopic Roux-en-Y versus mini-gastric bypass for the treatment of morbid obesity: a prospective randomized controlled clinical trial. Ann Surg 2005;242:20-8. [CrossRef]

5. Quan Y, Huang A, Ye M, Xu M, Zhuang B, Zhang P, et al. Efficacy of Laparoscopic Mini Gastric Bypass for Obesity and Type 2 Diabetes Mellitus: A Systematic Review and Meta-Analysis. Gastroenterol Res Pract 2015;2015:152852. [CrossRef]

6. Milone M, Lupoli R, Maietta P, Di Minno A, Bianco P, Ambrosino $P$, et al. Lipid profile changes in patients undergoing bariatric surgery: a comparative study between sleeve gastrectomy and mini-gastric bypass. Int J Surg 2015;14:28-32.

7. Yorke E, Sheppard C, Switzer NJ, Kim D, de Gara C, Karmali S, et al. Revision of sleeve gastrectomy to Roux-en-Y Gastric Bypass: A Canadian experience. Am J Surg 2017;213:970-4.

8. Gagner M, Buchwald JN. Comparison of laparoscopic sleeve gastrectomy leak rates in four staple-line reinforcement options: a systematic review. Surg Obes Relat Dis 2014;10:71323. [CrossRef]

9. Khoursheed M, Al-Bader I, Mouzannar A, Ashraf A, Bahzad Y, Al-Haddad A, et al. Postoperative Bleeding and Leakage After Sleeve Gastrectomy: a Single-Center Experience. Obes Surg 2016;26:3007. [CrossRef]

10. Cesana G, Cioffi S, Giorgi R, Villa R, Uccelli M, Ciccarese F, et al. Proximal Leakage After Laparoscopic Sleeve Gastrectomy: an Analysisof Preoperative and Operative Predictors on 1738 ConsecutiveProcedures. Obes Surg 2018;28:627-35. [CrossRef]

11. Usta $S$, Karabulut K. Effect of fibrin glue or suture on leakage in patients undergoinglaparoscopic sleeve gastrectomy. Niger J Clin Pract 2018;21:1209-12.

12. Abou Rached A, Basile M, El Masri H. Gastric leaks post sleeve gastrectomy: review of its prevention and management. World J Gastroenterol 2014;20:13904-10. [CrossRef]

13. Yardimci S, Coskun M, Demircioglu S, Erdim A, Cingi A. Is Concomitant Cholecystectomy Necessary for Asymptomatic Cholelithiasis During Laparoscopic Sleeve Gastrectomy? Obes Surg 2018;28:469-73. [CrossRef] 